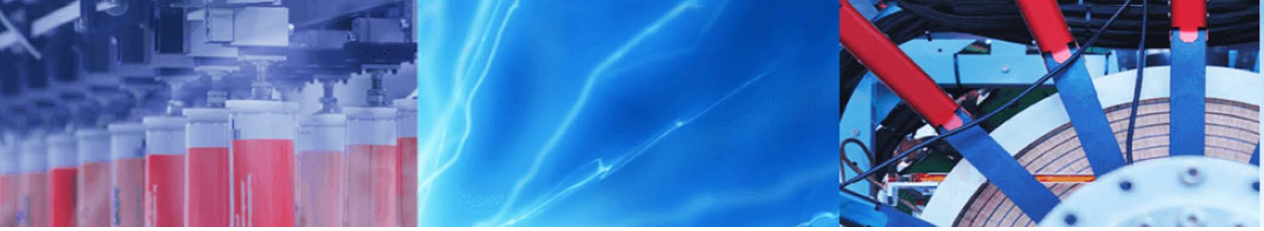

Research Article

\title{
Comparison of basic droop control with linear and nonlinear internal control of boost converters feeding resistive load
}

\author{
Sonia Moussa ${ }^{1}$ ] Manel Jebali Ben Ghorbal ${ }^{1} \cdot$ Ilhem Slama-Belkhodja ${ }^{1}$
}

Received: 21 October 2019 / Accepted: 8 January 2020 / Published online: 14 January 2020

(c) Springer Nature Switzerland AG 2020

\begin{abstract}
Droop control method is largely adopted to achieve load sharing among paralleled converters in standalone DC microgrid. However, this control is often associated with a lower layer of control performed using PI controllers. These PI controllers are used to control the inductor current and output voltage of the converters, although these latter being nonlinear systems and despite the variability of production and consumption on renewable sources-based DC microgrid. Hence, in this paper, the authors address the comparison of the application of basic droop control when the lower layer of control is performed using PI controllers or using nonlinear controllers. The comparison is applied to a system including two distributed generators interfaced to the DC bus by non-isolated boost converters. The modeling of the converter and the details of the synthesis of the different controllers are detailed. Simulation results are then presented using PSIM software.
\end{abstract}

Keywords Boost converter · Droop control · Current load sharing

\section{Introduction}

The proliferation on the market of small-scale renewable energy sources that generate electricity in DC form has more and more facilitated the development of off-grid solution for non-electrified rural regions in the world. In this sense, numerous DC home appliances are available on the market [1], greatly promoting residences with DC distribution system [2-5]. Coupled to this, the growing trend toward the use of all-electric vehicles will make the battery storage system more affordable with a better storage capacity. This will greatly contribute to the adoption of LVDC microgrid for residential application as presented in Fig. 1. This field is actually gathering the intention of many researchers, covering the branch of standardization $[6,7]$, control strategy $[8,9]$, power flow management [10,
11], operation stability [12-14], storage technologies and control $[15,16]$, and renewable source efficiency [17].

Regarding the control strategy, the first layer of hierarchical control concerns the current load sharing among the paralleled converters [8]. Often, error load sharing occurs when there is a mismatch of voltage values provided by the sources at the bus level. If the output voltage of each converter is well regulated, then this mismatch comes from the difference in cable impedance connecting the converters to the DC bus [18].

To overcome the error in current load sharing, some types of controls, such as active load sharing [19-22] and droop control $[4,5,8,23]$ have been proposed in the literature. If the active load sharing method presents the advantages of being simple to implement with a good performance, its major drawbacks consist on the need of a large bandwidth communication link and its

Sonia Moussa, sonia.moussa@enit.utm.tn; Manel Jebali Ben Ghorbal, manel.jebalibenghorbal@enit.utm.tn; Ilhem Slama-Belkhodja, ilhem.slamabelkhodja@enit.utm.tn | 'LR11ES15 Laboratoire des Systèmes Electriques, Ecole Nationale d'Ingénieurs de Tunis, Université de Tunis El Manar, 1002 Tunis, Tunisia. 


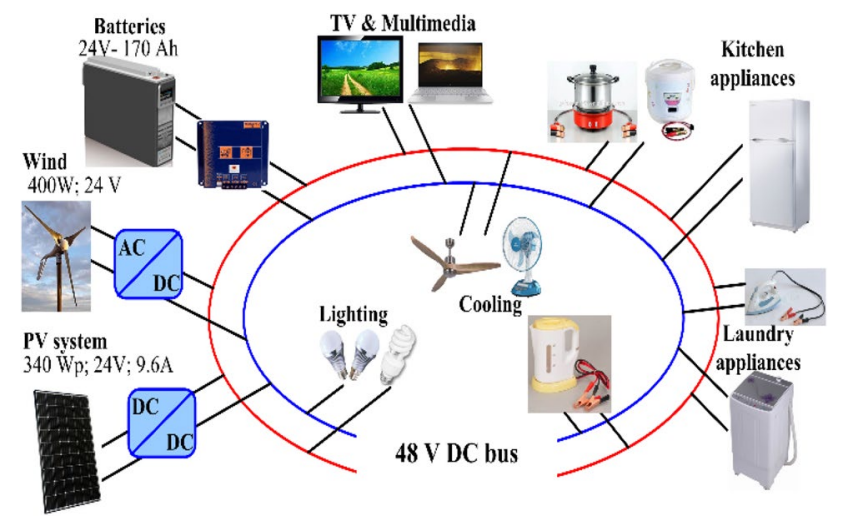

Fig. 1 Residential DC microgrid

low flexibility and modularity. In fact, a higher bandwidth communication link is needed with the increasing number of distributed generators present in the microgrid. Droop control, on the other hand, presents a poorer current load sharing capacity compared to the active load sharing method, but it does not need a communication link for its implementation and the modularity of the system elements is not affected in the case of an add or removal of distributed generators. Eventually, a low-bandwidth communication link is enough in case of a droop control with voltage restoration.

Hence in this paper, droop control is chosen as load sharing method. The implementation of droop control consists in controlling each converter in parallel individually first, and then adding an external loop which ensure the load sharing among the paralleled converters. Generally, the internal control of the converters in the droop control method found in the literature use PI controllers [4, 5, 8, 23-25], though the converters are nonlinear system. Moreover, for a renewable-based DC microgrid, the intermittency of the sources will lead to the variability of the energy production. The same goes for the load variation that depends on the user's needs, in a residential application. In this case, the use of PI controller may not be robust when a large range of operating points is considered. Therefore, this paper proposes to compare the performance of the implementation of a basic droop control when the internal control of the converter is performed via PI controllers or nonlinear controllers.

The paper is structured as follows: a presentation of the studied system, detailing the power stage modeling, the controller synthesis, and droop control application, is presented in Sect. 2. Section 3 discusses the simulation results while Sect. 4 gives a conclusion of the paper.

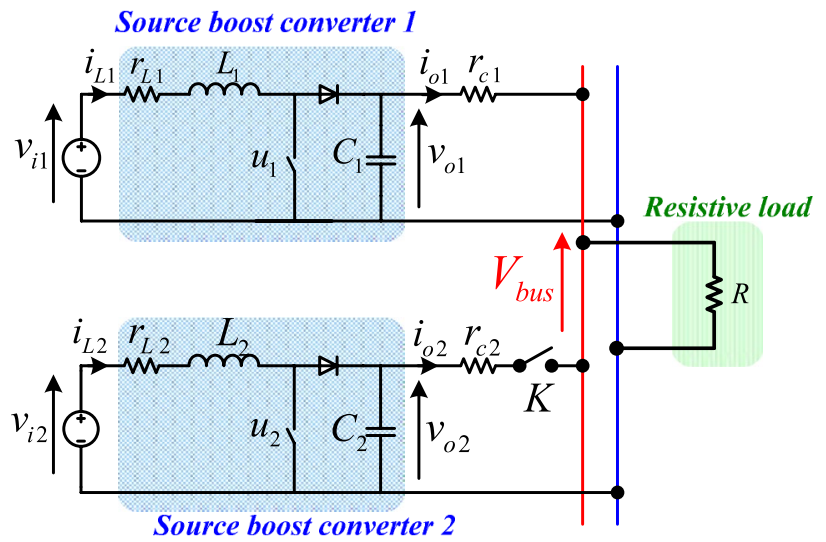

Fig. 2 Case-study system

\section{Studied system presentation}

\subsection{Power stage model}

As the interest is laid on the parallel operating of distributed generators, we consider the system presented in Fig. 2. The studied system is composed of two distributed generators with their interfacing converter and a resistive load. Non-isolated boost converter is taken as interfacing converter. The DC bus level chosen for application is $48 \mathrm{~V}$, while the input voltages of the sources are inferior to this value. This choice of voltage level has been discussed in [1]. The resistances $\mathrm{rc} 1$ and $\mathrm{rc} 2$ represent the cable resistance connecting each converter to the bus.

The power loss in the inductor of the converters is taken into account since it presents a great impact on the converter output voltage. This loss is portrayed by the resistances $r_{L 1}$ and $r_{L 2}$. The large signal average model system of equations governing each converter is given by (1), where the subscript $k$ is related to each converter.

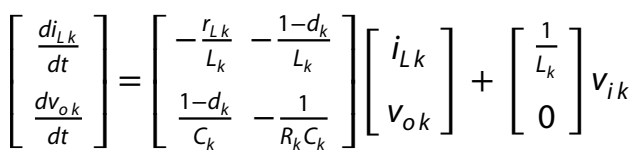

In (1), $d_{k}$ represents the duty cycle associated with the $k$ th converter and $R_{k}$ represents the load resistance for a given power consumed $P$ by the converter and is expressed by

$R_{k}=\frac{v_{o k}^{2}}{P_{o k}}$

Since linear controller will be considered later, the small signal average model describing the boost converter is given by (3) 


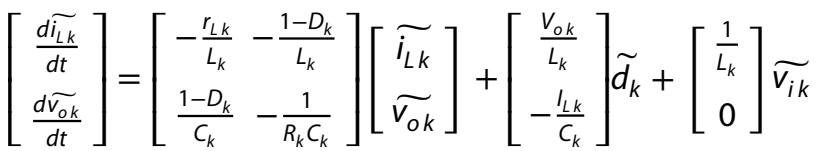

This small signal average model is obtained by a linearization of (1) around an operating point of the converter. Each variable of (1) is defined as a sum of an average value and a small variation around this average value, as expressed in (4).

$i_{L k}=I_{L k}+\widetilde{i_{L k}}$

$v_{o k}=V_{o k}+\widetilde{v_{o k}}$

$d_{k}=D_{k}+\tilde{d}_{k}$

$v_{i k}=v_{i k}+\widetilde{v_{i k}}$

Equations governing the power stage being defined, the synthesis of each controller type is then detailed in the following subsection $\mathrm{B}$.

\subsection{Controller synthesis}

For the controller synthesis of the converter intern variable, Proportional-Integral regulator is first considered

\subsubsection{Linear PI controller synthesis}

The PI controller synthesis goes through the description of the transfer function of the variable to be controlled. Since in our case, the inductor current and the output voltage of the converter are both controlled, a cascaded control loop is adopted. The block diagram illustration the control is presented in Fig. 3.

Since cascaded loop is used, the inner current loop dynamic must be faster than the outer voltage loop.

By using the Laplace transform of (3), and by considering $\widetilde{v_{i k}}$ as a perturbation, the control-to-current transfer function $G_{i d}$ is expressed by (5).

$G_{i d}(s)=\frac{\tilde{i_{L k}}}{{\tilde{d_{k}}}_{k}}=\frac{V_{o k}}{s L_{k}+r_{L k}}$

This expression is obtained while neglecting the term in $\widetilde{v_{o k}}$ of the Laplace transform of (3), since the dynamic of the outer loop is considered slow compared to inner loop one.
Current-to-output voltage transfer function case is obtained by assuming the inductor current equal to its reference after a transient in the current loop. Since the inner loop is much faster than the outer loop, the perturbation in the duty cycle can be neglected. Therefore, the current-to-output voltage transfer function expression is given by (6).

$G_{v i}(s)=\frac{\widetilde{v_{o k}}}{\widetilde{i_{L k}}}=\frac{\left(1-D_{k}\right)}{s C_{k}+\frac{1}{R_{k}}}$

The expression of the $\mathrm{PI}$ controller associated to each loop is then given by (7) and (9) respectively for the current and voltage controllers.

$C_{i k}(s)=K_{p i k}+\frac{K_{i i k}}{s}$

$C_{v k}(s)=K_{p v k}+\frac{K_{i v k}}{s}$

As the voltage and current loop transfer functions are 1 st order ones, the controller synthesis is done using pole compensation method.

\subsubsection{Nonlinear controller synthesis}

Similar to the synthesis of the linear controller, a cascaded controller as in [12] is also adopted. The control of the inductor current, the inner loop control, is realized by sliding mode control while the outer loop voltage control is done by flatness-based control. The schematic block diagram depicting the control is presented in Fig. 4.

The sliding surface $S_{k}$ of the sliding mode controller and the condition on its derivative is given in (9).

$S_{k}=i_{L k}-i_{L k r e f}+K_{i k} \int\left(i_{L k}-i_{L k r e f}\right) d \tau$

$\dot{S}_{k}=-\lambda_{k} S_{k}$

From (9), one can draw the duty cycle's expression that is given by (10).

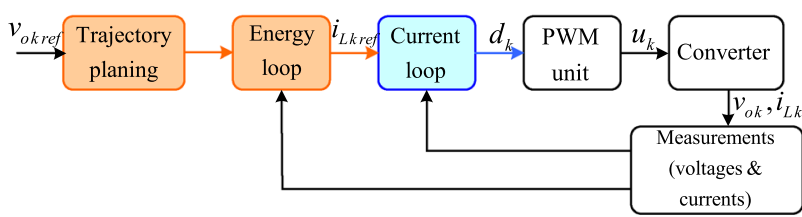

Fig. 4 Nonlinear control block diagram

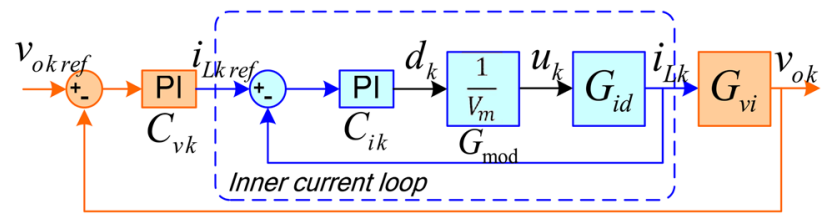

Fig. 3 Linear control block diagram 


$$
\begin{aligned}
& d_{k}=\frac{1}{v_{o k}}\left(a_{k}+b_{k}\right) \\
& a_{k}=v_{o k}+r_{L k} i_{L k}-v_{i k} \\
& b_{k}=L_{k}\left[\left(K_{i k}+\lambda_{k}\right)\left(i_{L k r e f}-i_{L k}\right)+K_{i k} \lambda_{k} \int\left(i_{L k r e f}-i_{L k}\right)\right]
\end{aligned}
$$

For the flatness-based control of the outer loop, the energy of the output voltage capacitor is taken as flat output candidate $y$. its expression is given by (11):

$y=\frac{1}{2} C_{k} v_{o k}^{2}$

A power balance of the converter is then realized in order to establish the relation between the control variable $i_{L k}$ and the flat output candidate $y$. we assume that the power induced by the variation of the magnetic energy is negligible and the current loop dynamic is much faster than the outer loop.

$\dot{y}=P_{i n}-r_{L k} i_{L k}^{2}-P_{o k}=P_{i n}-r_{L k}\left(\frac{P_{i n}}{v_{i k}}\right)^{2}-P_{o k}$

The current control is then obtained by resolving (12) and is expressed by (13).

$i_{L k}=\frac{P_{i n}}{v_{i k}}=\frac{2 P_{i \max }}{v_{i k}}\left(1-\sqrt{1-\frac{\dot{y}+P_{o k}}{P_{i \max }}}\right)$

With $P_{i \max }=\frac{v_{i k}^{2}}{4 r_{L k}}:$ maximum transmissible power

$P_{o k}=v_{o k} i_{o k}:$ output power

The control law adopted for the energy of the output capacitor is given by

$\left(\dot{y}-\dot{y}_{r e f}\right)+K_{p y}\left(y-y_{r e f}\right)+K_{i y} \int\left(y-y_{r e f}\right) d \tau=0$

Thus, the expression of $\dot{y}$ in (13) is deduced from (14) and expressed as follows:

$\dot{y}=\dot{y}_{r e f}+K_{p y}\left(y_{r e f}-y\right)+K_{i y} \int\left(y_{r e f}-y\right)$

The nonlinear controller block diagram is thus illustrated in Fig. 5.

\subsection{Droop control with voltage restoration}

In order to simplify the comprehension of the droop control principle, let us consider the output voltage $v_{o k}$ of each converter well-regulated and assimilated to a voltage source as shown in Fig. 6 . The droop control consists

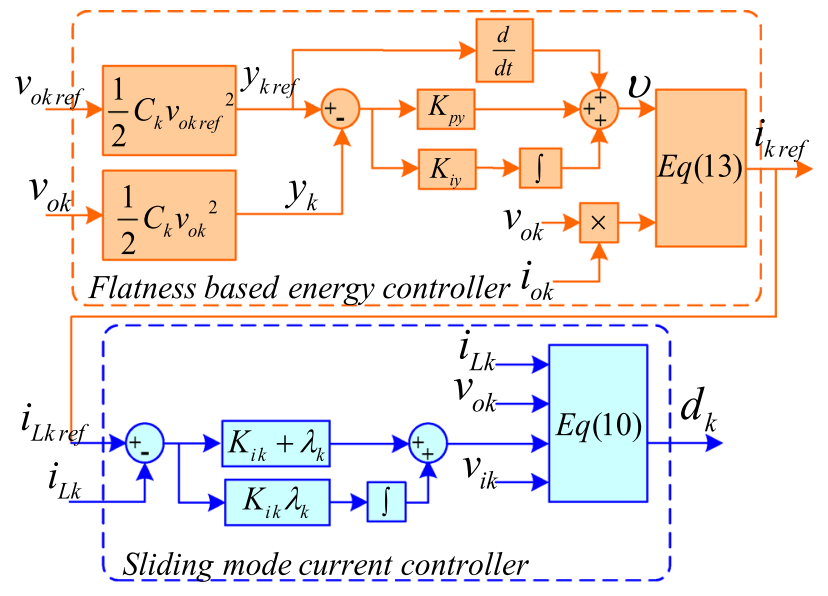

Fig. 5 Nonlinear controller block diagram

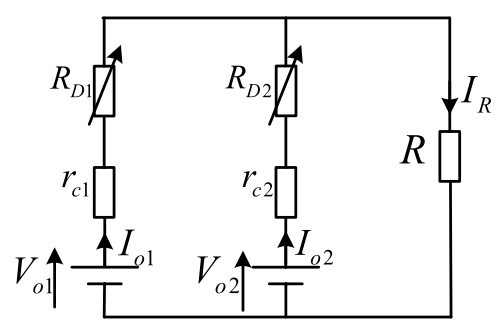

Fig. 6 Droop control principle

on emulating virtual impedance $R_{D k}$ in each branch containing a source, so that having the same voltage value on the bus. This feat is performed by using an outer loop with a droop gain, that is subtracted to the control loop of the converter.

The reference $V_{o \text { kref }}$ of the output voltage of the converter then becomes as expressed in (16), $V_{\text {ref } k}$ being the fixed value of the desired bus voltage, $R_{D k}$ the virtual droop resistance and $i_{o k}$ the output current of the converter.

$V_{\text {ok ref }}=V_{\text {ref } k}-R_{D k} i_{o k}$

Considering the maximum output voltage deviation $\varepsilon_{v}$ and the maximum output current $I_{\text {ok max }}$ of the converter, the virtual resistance $R_{D k}$ is calculated as in (17).

$R_{D k}=\frac{\varepsilon_{v}}{I_{o k \max }}$

From (16), one can deduce that the output voltage of the converter will be lower than the desired reference, due to the virtual resistance. Hence, another outer loop that allows restoring the bus voltage, according to the drop occasioned by the virtual resistance, is added to the overall control. Finally, the output voltage reference of 
the converter is given by (18), where $\delta v_{o k}$ represents the processed voltage deviation.

$V_{\text {ok ref }}=V_{\text {ref } k}-R_{D k} i_{o k}+\delta v_{o k}$

The processed voltage deviation $\delta v_{o k}$ results from the compensation through PI regulator of the sensed bus voltage $V_{\text {bus }}$ and the desired bus voltage $V_{\text {bus ref. }}$.

The outer loop control to be added to the control of the internal variable of the converter is then depicted in Fig. 7.

\section{Simulations and discussions}

In order to compare the performance of the droop control combined with the two types of control for the internal variable of the converters, three scenarios will be considered. Given that each converter is sized to operate within

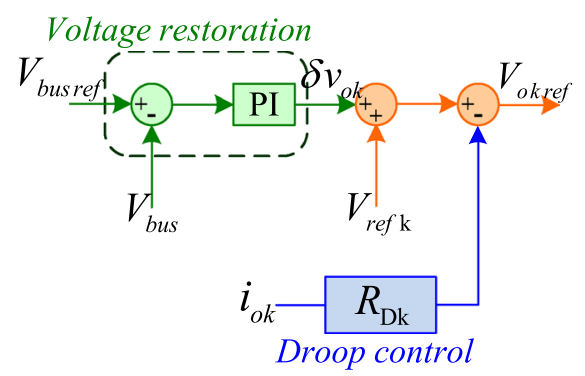

Fig. 7 Droop control with voltage restoration
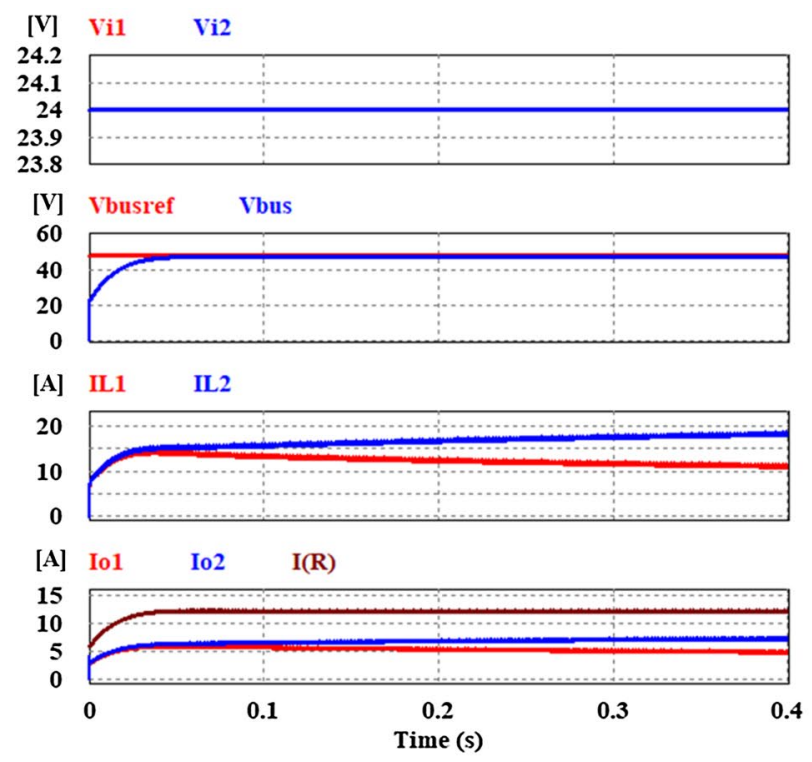

(a) Without droop control a maximum power of $400 \mathrm{~W}$, the load power range during the operating of two converters will then be within $800 \mathrm{~W}$.

For the first two scenarios, the purpose is to compare the load sharing capabilities of the system depending on the type of the internal control adopted. A resistance of $3.84 \Omega$ is taken as load, that corresponds to a consumed power of $600 \mathrm{~W}$. The cable resistance linking the first converter to the bus is the double of the one linking the second converter. And both converters have the same characteristics. In the first scenario (scenario 1), the input voltages of both converters are the same while in the second scenario (scenario 2) the input voltages of the converters are different. For each scenario, the evolution of the voltage and current with and without droop control is shown.

For the third scenario (scenario 3), the purpose is to compare the plug and play capability of the system. A load resistance of $7.68 \Omega$ is considered, it corresponds to a consumed power of $300 \mathrm{~W}$. The first converter's cable resistance is still the double of the second converter's one. The input voltages of both converters are identic. Both converters are first initially connected. The second converter is then disconnected at $t=0.2 \mathrm{~s}$ and reconnected at $t=0.4 \mathrm{~s}$ by the means of the switch $K$ in Fig. 2 .

Figures 8 and 9 show the simulation results of the first scenario when adopting linear and nonlinear controllers respectively, while Fig. 10 highlights the behavior of the droop controlled-system in steady-state.

As can be seen in Figs. 8a and 9a, despite the converters having the same characteristics and the same operating point the one connected to the bus through a lower cable
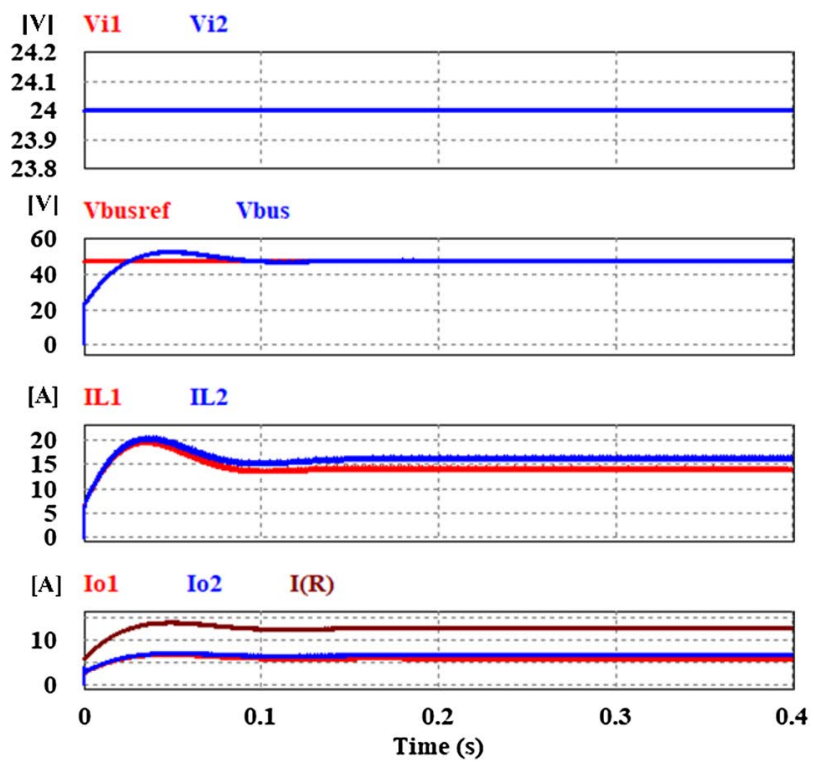

(b) With droop control

Fig. 8 Scenario 1 with PI controllers 


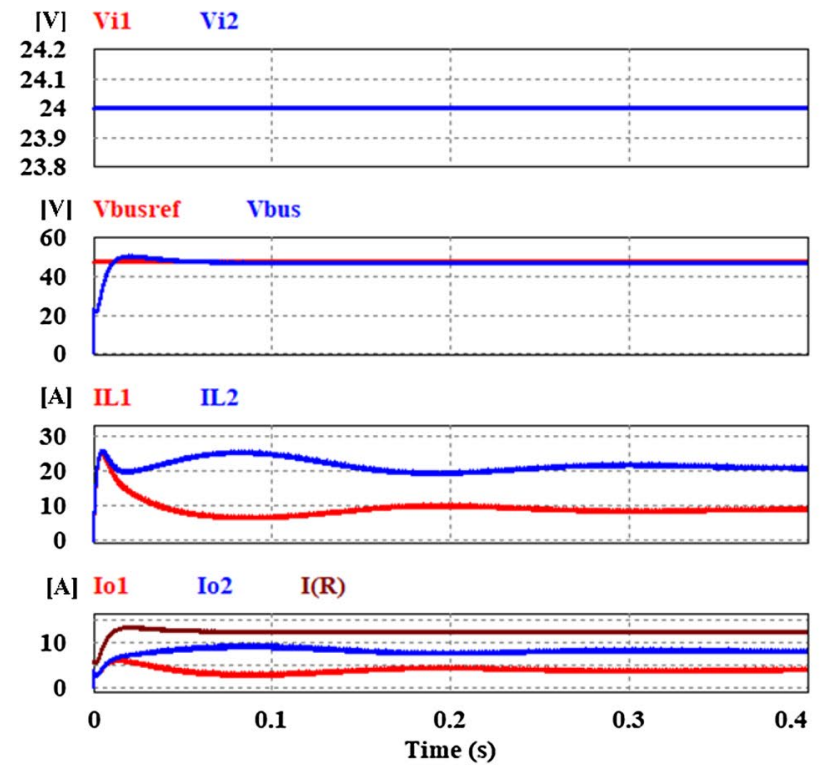

(a) Without droop control

Fig. 9 Scenario 1 with nonlinear controllers

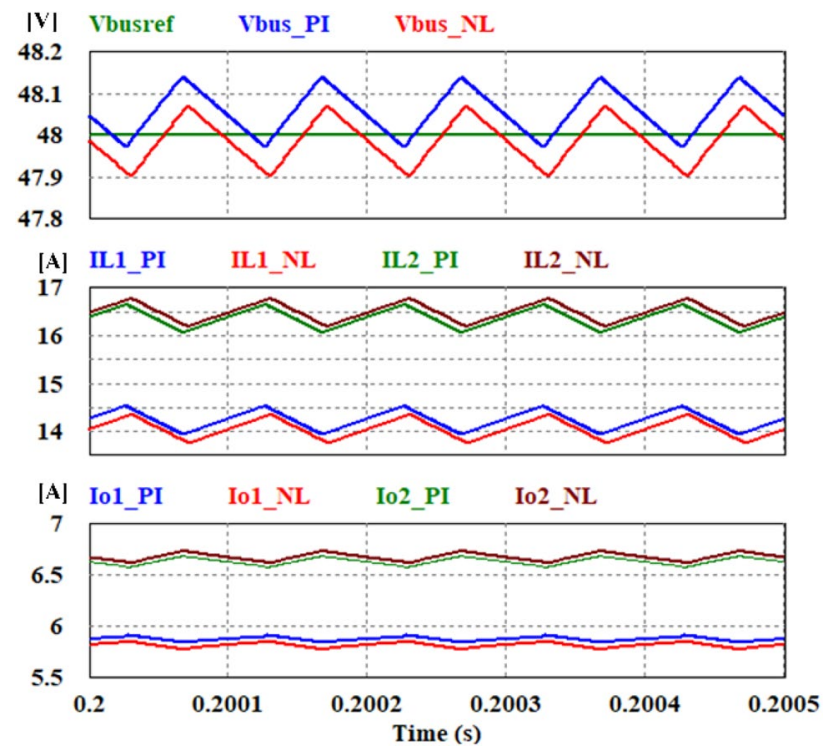

Fig. 10 Zoom scenario 1 for droop control in steady-state

resistance, here the converter 2 , have to produce more current to the load.

The error in current load sharing is slightly worse for the nonlinear controlled-system compared to the one linearcontrolled. However, after applying the droop control as presented in Figs. $8 \mathrm{~b}$ and 9b, the error load sharing is minimized. The transient of the nonlinear-controlled system is faster than the one of the linear-controlled one, but it has a high inrush current.
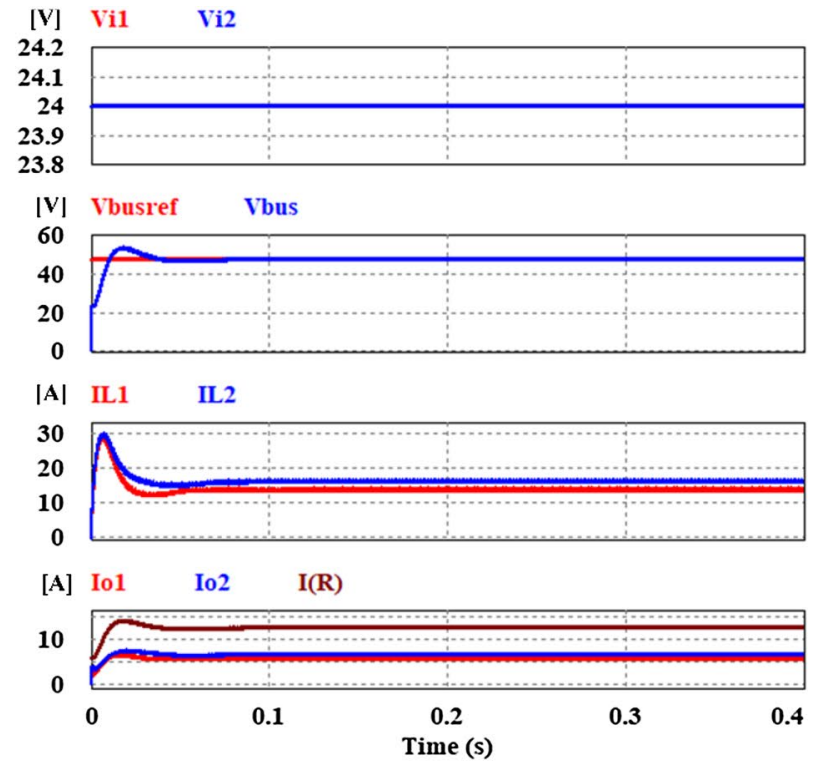

(b) With droop control

One the other hand, during the steady-state operation as shown in Fig. 10, the current load sharing is almost identical whether the system is internally controlled in a linear or nonlinear way. Nonetheless, the bus regulation of the nonlinear-controlled system is better.

The second scenario simulation results are given in Figs. 11 and 12, respectively, when adopting linear and nonlinear controllers. Likewise in the first scenario, the zoom operation in steady-state while droop control is applied is presented in Fig. 12.

Despite the input voltage of converter 2 is greater than the one of converter 1, the error load sharing remains, as can be seen in Figs. 11a and 12a. The converter with the lower cable resistance is still overworked. The application of droop control minimizes the error load sharing among the converters as shown in Figs. $11 \mathrm{~b}$ and $12 \mathrm{~b}$. The system controlled non-linearly is still faster with high inrush current. However, the zoom in steady-state operation presented in Fig. 12 shows an almost identical current sharing and voltage regulation for both linear and nonlinear controlled system (Fig. 13).

The third scenario simulation results are depicted in Figs. 14 and 15. The results when PI controllers are adopted are presented in Figs. 14a and 15a while the ones with nonlinear controllers are presented in Figs. 14b and 15b. During the disconnection and reconnection of the second converter, the transient of the nonlinear controlled system is faster and more stable than the one of the linear controlled system. The difference in rapidity is especially significant during the reconnection. As can be seen in 

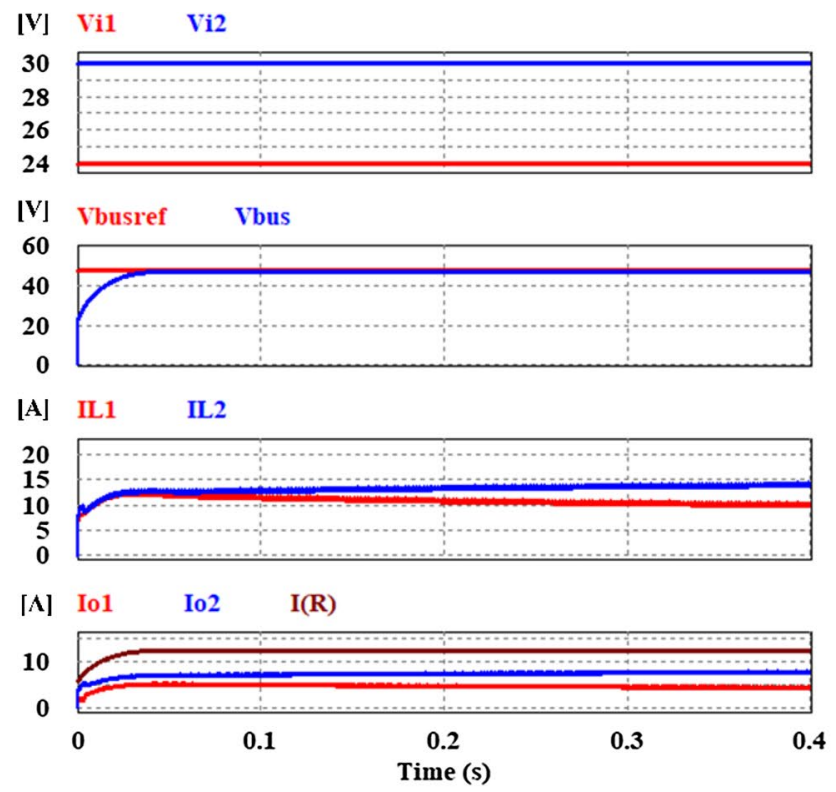

(a) Without droop control

Fig. 11 Scenario 2 with PI controllers
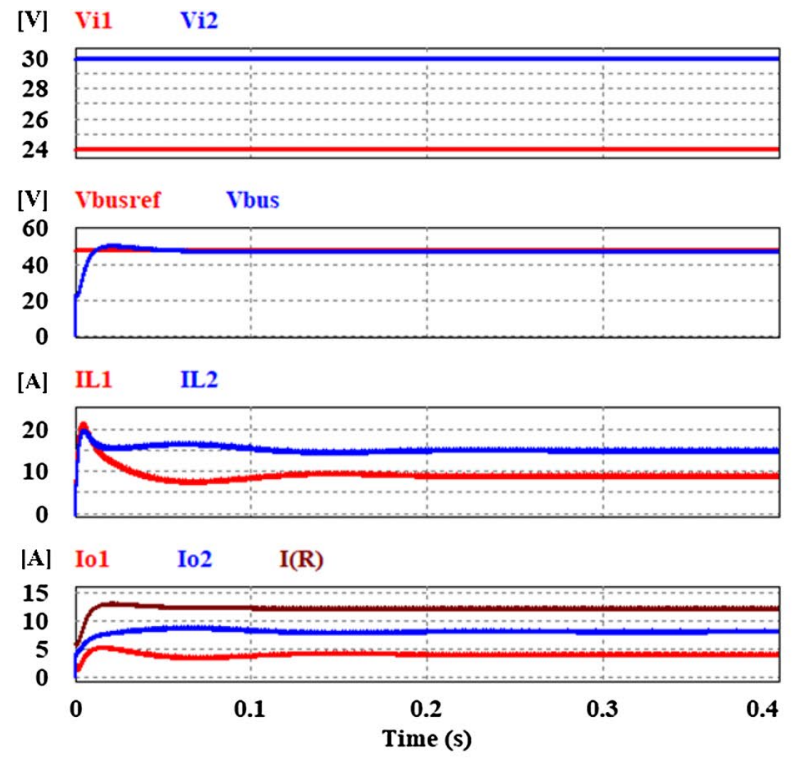

(a) Without droop control

Fig. 12 Scenario 2 with nonlinear controllers

Fig. 14, the PI controlled system needs $325 \mathrm{~ms}$ to attain stable operation while the nonlinear controlled one needs only $50 \mathrm{~ms}$. The latency time during the reconnection is equally much slower for the PI controlled system, compared to the nonlinear one, with a latency time of $13 \mathrm{~ms}$ against $1.3 \mathrm{~ms}$ respectively.

On the other hand, during the disconnection of the second converter, the nonlinear controlled system
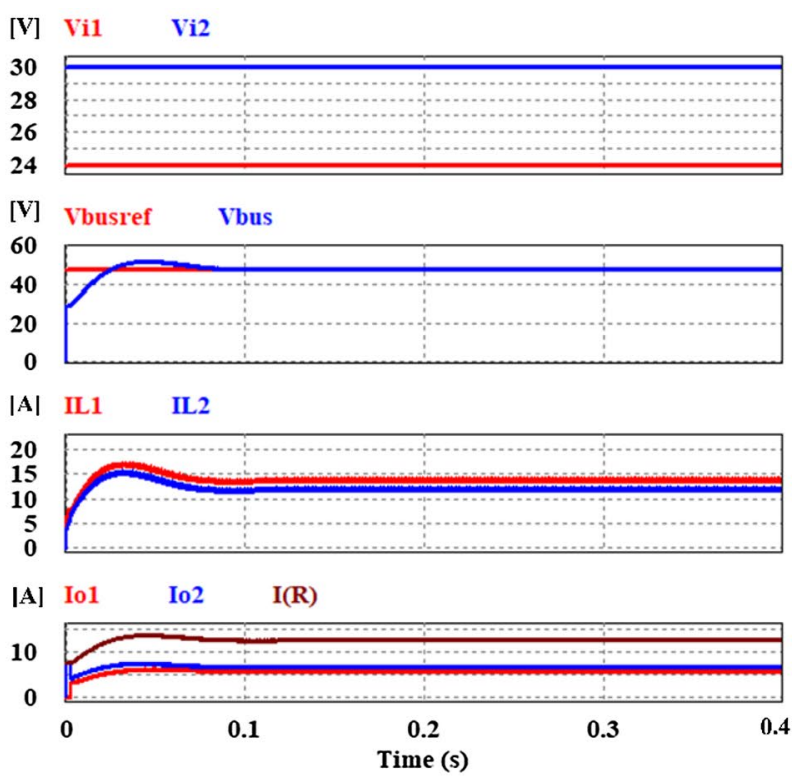

(b) With droop control
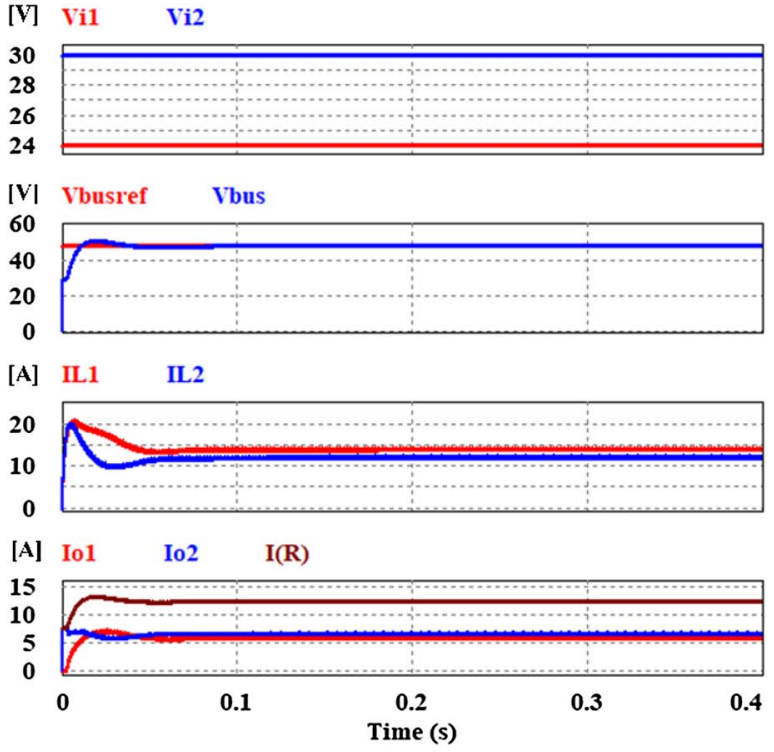

(b) With droop control

almost instantaneously regains stable operation while the $\mathrm{PI}$ controlled one needs $80 \mathrm{~ms}$ to regain stable operation.

Furthermore, the overshoot in the output voltage and inductor current during the disconnection and reconnection are equally important on the PI controlled system, while it is negligible on the nonlinear controlled system. 


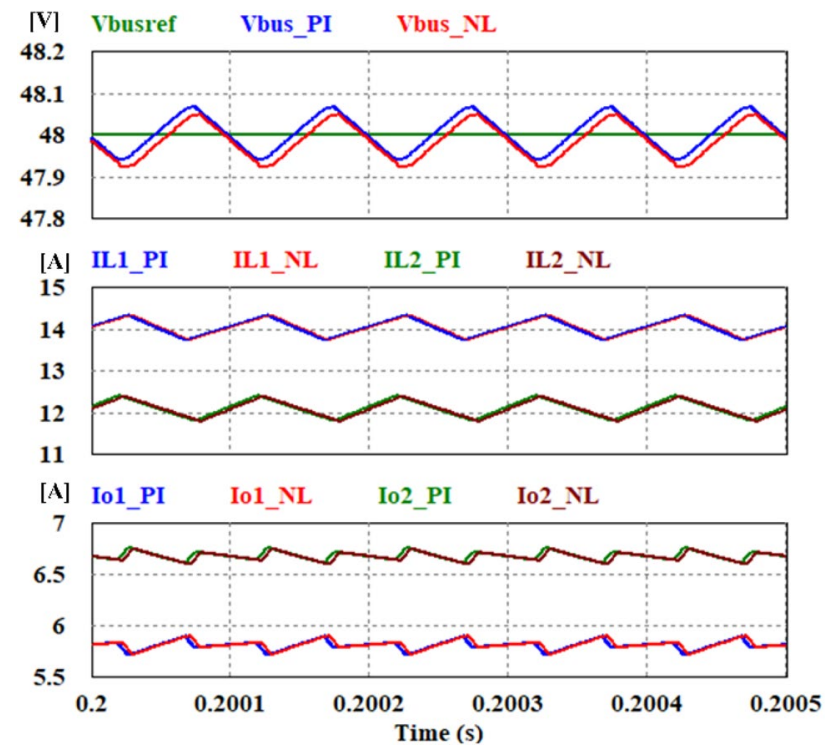

Fig. 13 Zoom scenario 2 for droop control in steady state

\section{Conclusion}

Droop control method is largely adopted to enhance load sharing among paralleled converters in DC microgrid, thanks to its flexibility and modularity. This control is generally associated with PI controllers for the internal control of the output voltage and inductor current of the converter. However, for a renewable source based residential DC microgrid, energy production and load variability are unavoidable. Hence, with a system operating within a wide range operating points, $\mathrm{PI}$ controllers may not be robust enough.

Thus, in this paper, the parallel operation of two boost converters feeding a resistive load is investigated and compared considering a linear and nonlinear control of the internal variables of these converters. The cable resistances linking the converters to the DC bus are taken different and it has been shown that a non-negligible error load sharing occurs among the converters, whether the input voltages of the converters are identical or different in the case that the converters are paralleled without load sharing scheme. The load sharing capability of the system is compared through the first two scenarios. It has been shown that the application of droop control on the system gives almost identical results in steady-state operation, though the nonlinear-controlled system is faster with a higher inrush current at the initial time, compared to the linear-controlled one. The plug-and-play capability has been investigated through the third scenario. The results show that the nonlinear controlled system offers a faster and more stable performance during the transient compared to the linear controlled system.

In perspective, the application of droop control with the same system while considering a constant power load type will be investigated later.

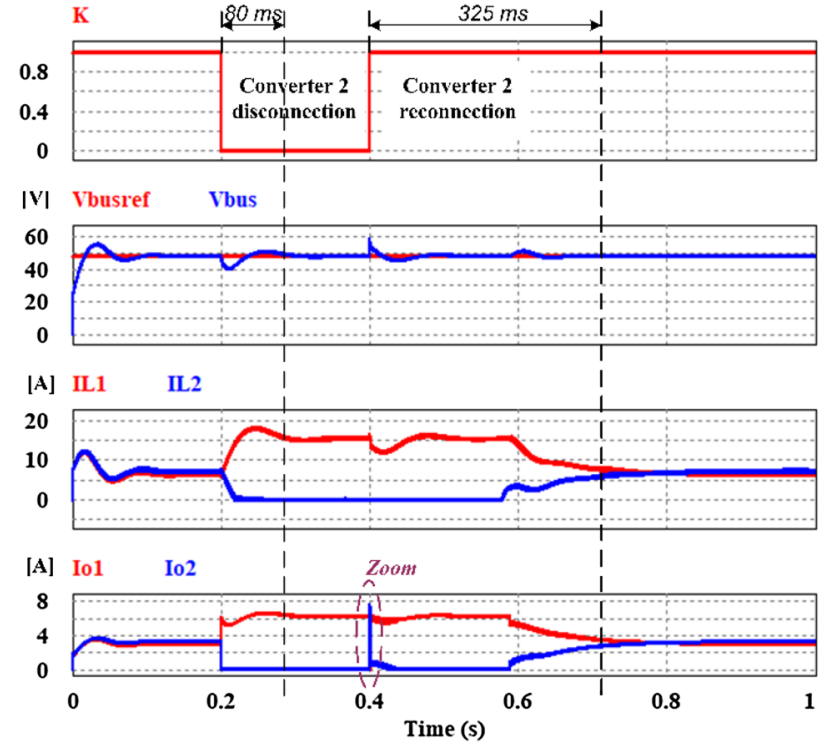

(a) With PI controllers

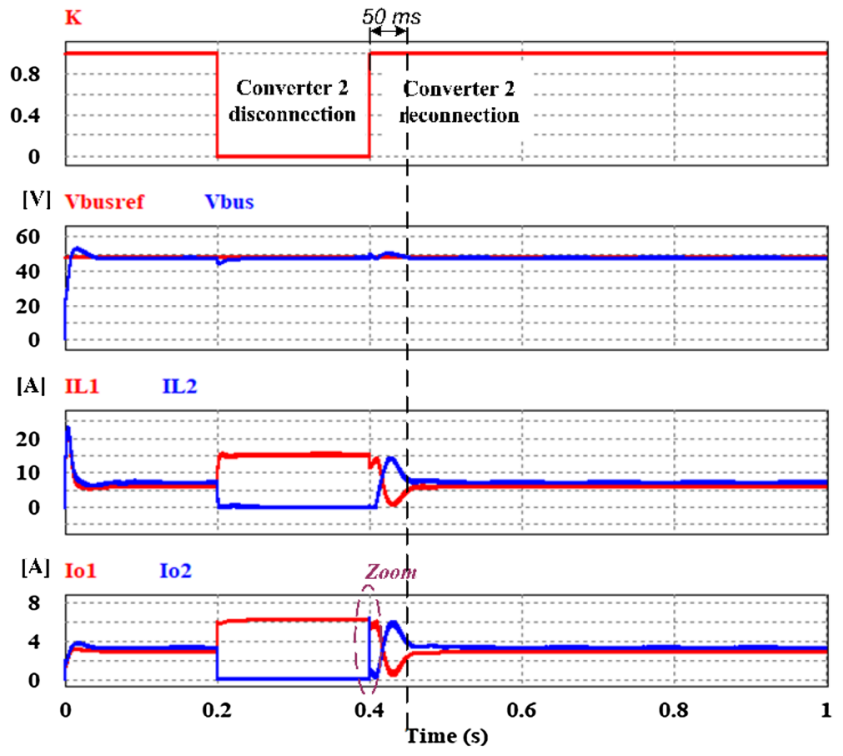

(b) Without nonlinear controllers

Fig. 14 Disconnection and reconnection of source 2 


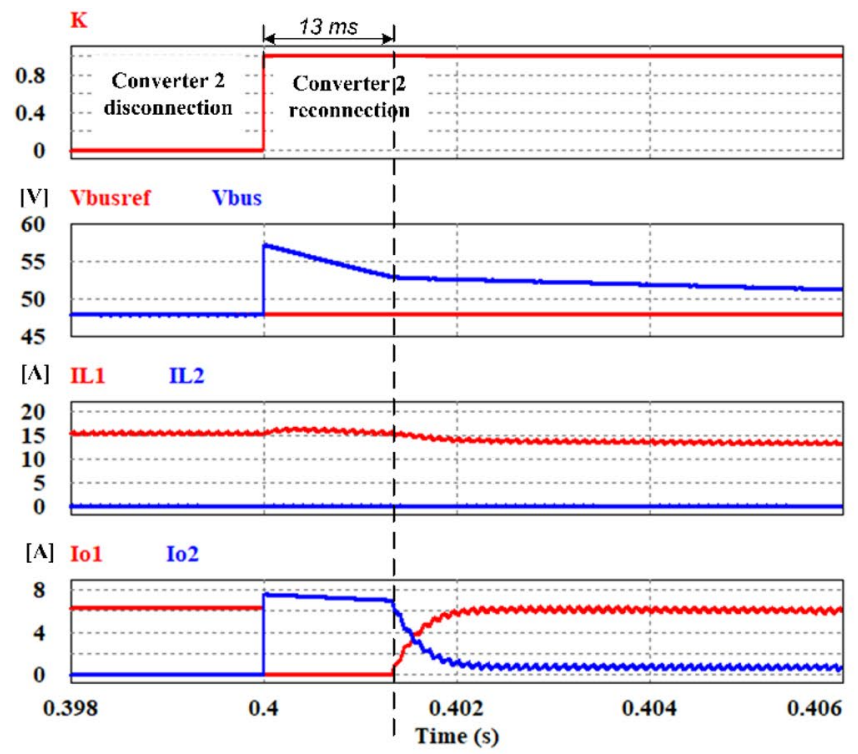

(a) With PI controllers

Fig. 15 Zoom during reconnection of one source

Acknowledgements This work was supported by the Tunisian Ministry of High Education and Research under Grant LSE-ENIT-LR $11 \mathrm{ES} 15$ and funded in part by NAS and USAID under the USAID Prime Award Number AID-OAA-A-11-00012. Any opinions, findings, conclusions, or recommendations expressed in this article are those of the authors alone, and do not necessarily reflect the views of USAID or NAS.

\section{Compliance with ethical standards}

Conflict of interest The authors declare that they have no conflict of interest.

\section{References}

1. Moussa S, Jebali Ben Ghorbal M, Slama-Belkhodja I (2019) Bus voltage level choice for standalone residential DC nanogrid. Sustain Cities Soc 46:101431

2. Taufik T, Muscarella M (2016) Development of DC house prototypes as demonstration sites for an alternate solution to rural electrification. In: 2016 6th international annual engineering seminar (InAES), pp 262-265

3. Castillo-Calzadilla T, Macarulla AM, Kamara-Esteban O, Borges CE (2018) Analysis and assessment of an off-grid services building through the usage of a DC photovoltaic microgrid. Sustain Cities Soc 38:405-419

4. Golshannavaz S, Mortezapour V (2018) A generalized droop control approach for islanded DC microgrids hosting parallelconnected DERs. Sustain Cities Soc 36:237-245

5. Ghalebani P, Niasati M (2018) A distributed control strategy based on droop control and low-bandwidth communication in DC microgrids with increased accuracy of load sharing. Sustain Cities Soc 40:155-164

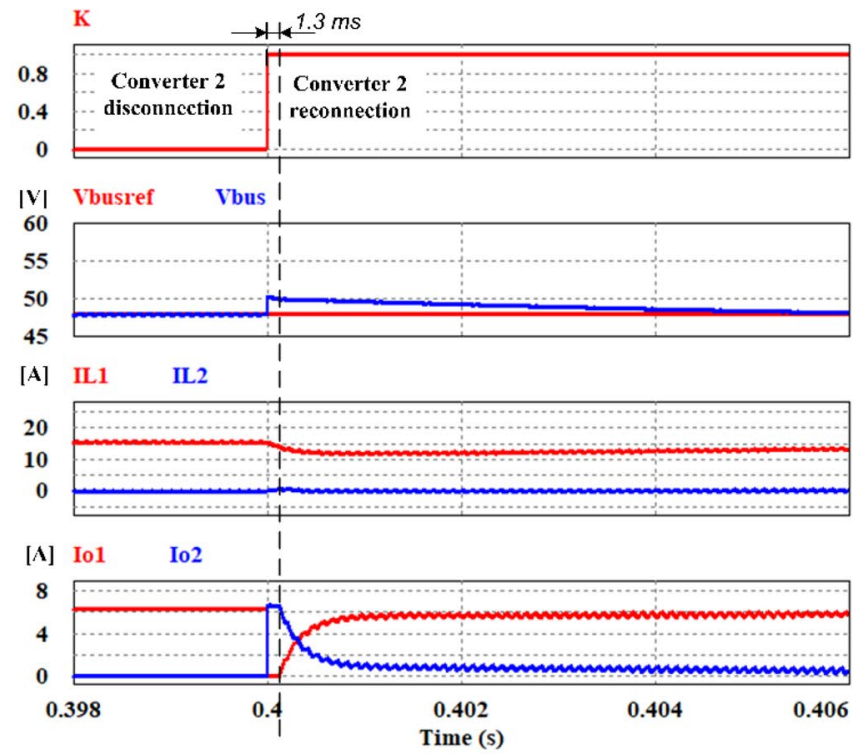

(b) Without nonlinear controllers

6. Decuir J, Michael P (2017) Draft IEEE standard for DC microgrids for rural and remote electricity access applications. In: 2017 IEEE conference on technologies for sustainability (SusTech), pp 1-5

7. Kaipia T et al (2017) Survey of market prospects and standardisation development needs of LVDC technology. CIRED Open Access Proc J 2017(1):454-458

8. Augustine S, Mishra MK, Lakshminarasamma N (2015) Adaptive droop control strategy for load sharing and circulating current minimization in low-voltage standalone DC microgrid. Sustain Energy IEEE Trans 6(1):132-141

9. Cornea O, Andreescu G, Muntean N, Hulea D (2017) Bidirectional power flow control in a DC microgrid through a switchedcapacitor cell hybrid DC-DC converter. IEEE Trans Ind Electron 64(4):3012-3022

10. Chen XY, Jin JX (2016) Energy efficiency analysis and energy management of a superconducting LVDC network. IEEE Trans Appl Supercond 26(7):1-5

11. Rodriguez-Diaz E, Palacios-Garcia EJ, Anvari-Moghaddam A, Vasquez JC, Guerrero JM (2017) Real-time energy management system for a hybrid AC/DC residential microgrid. In: 2017 IEEE second international conference on DC microgrids (ICDCM), pp 256-261

12. Gavagsaz-Ghoachani R, Saublet LM, Martin JP, Nahid-Mobarakeh B, Pierfederici S (2017) Stability analysis and active stabilization of DC power systems for electrified transportation systems, taking into account the load dynamics. IEEE Trans Transp Electrification 3(1):3-12

13. Herrera L, Zhang W, Wang J (2017) Stability analysis and controller design of DC microgrids with constant power loads. IEEE Trans Smart Grid 8(2):881-888

14. Su M, Liu Z, Sun Y, Han H, Hou X (2018) Stability analysis and stabilization methods of DC microgrid with multiple parallelconnected DC-DC converters loaded by CPLs. IEEE Trans Smart Grid 9(1):132-142

15. Weaver WW, Robinett RD, Parker GG, Wilson DG (2015) Energy storage requirements of dc microgrids with high penetration renewables under droop control. Int J Electr Power Energy Syst 68:203-209 
16. Mahjoubi C, Olivier J-C, Skander-mustapha S, Machmoum M, Slama-belkhodja I (2019) An improved thermal control of open cathode proton exchange membrane fuel cell. Int J Hydrog Energy 44(22):11332-11345

17. Triki-Lahiani A, Bennani-Ben Abdelghani A, Slama-Belkhodja I (2018) Partial shading effect investigation on PV performance indicators. In: 2018 9th international renewable energy congress (IREC), pp 1-6

18. Moussa S, Jebali Ben Ghorbal M, Slama-Belkhodja I (2017) DC load modelling and droop control analysis for autonomous residential DC microgrid. Presented at the ELECTRIMACS 2017, Toulouse, France, pp 1-6

19. Kamalesh ME, Vikashini M, Pradeep S (2018) Precompensated master slave control of parallel DC-DC converter in DC-microgrid. In 2018 international conference on current trends towards converging technologies (ICCTCT), pp 1-5

20. Raj A, Arulgandhi N, Patha L, Bhaskar DV (2018) Fuzzy logic based master-slave controller for paralleling DC-DC converters in LED applications. In: 2018 international conference on power energy, environment and intelligent control (PEEIC), pp 676-682

21. Chen S-Y, Yang B-C, Pu T-A, Chang C-H, Lin R-C (2019) Active current sharing of a parallel DC-DC converters system using bat algorithm optimized two-DOF PID control. IEEE Access 7:84757-84769
22. Zhang Z, Zhang Z, Xie S, Yang C (2016) A control strategy for paralleled bi-directional DC-DC converters used in energy storage systems. In: 2016 IEEE energy conversion congress and exposition (ECCE), pp 1-6

23. Khorsandi A, Ashourloo M, Mokhtari H, Iravani R (2016) Automatic droop control for a low voltage DC microgrid. Transm Distrib IET Gener 10(1):41-47

24. Nasirian V, Davoudi A, Lewis FL, Guerrero JM (2014) Distributed adaptive droop control for DC distribution systems. IEEE Trans Energy Convers 29(4):944-956

25. Lu X, Guerrero JM, Sun K, Vasquez JC (2014) An improved droop control method for DC microgrids based on low bandwidth communication with DC bus voltage restoration and enhanced current sharing accuracy. IEEE Trans Power Electron 29(4):1800-1812

Publisher's Note Springer Nature remains neutral with regard to jurisdictional claims in published maps and institutional affiliations. 